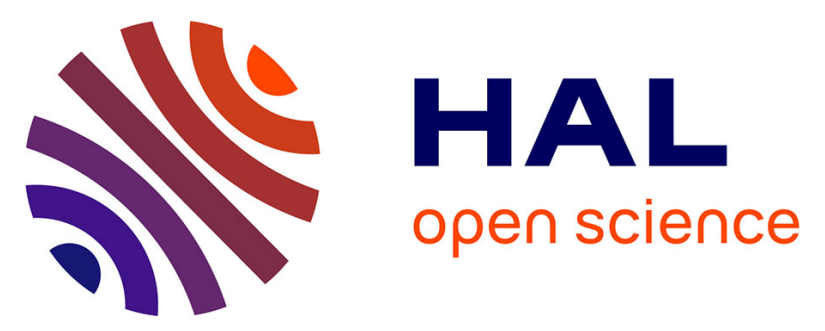

\title{
Numerical study of the influence of flux creep and of thermal effect on dynamic behaviour of magnetic levitation systems with a high- superconductor using control volume method
}

L. Alloui, F. Bouillault, S.M. Mimoune

\section{To cite this version:}

L. Alloui, F. Bouillault, S.M. Mimoune. Numerical study of the influence of flux creep and of thermal effect on dynamic behaviour of magnetic levitation systems with a high- superconductor using control volume method. European Physical Journal: Applied Physics, 2009, 45 (2), pp.1. 10.1051/epjap/2009008 . hal-00480144

\section{HAL Id: hal-00480144 \\ https://hal.science/hal-00480144}

Submitted on 3 May 2010

HAL is a multi-disciplinary open access archive for the deposit and dissemination of scientific research documents, whether they are published or not. The documents may come from teaching and research institutions in France or abroad, or from public or private research centers.
L'archive ouverte pluridisciplinaire HAL, est destinée au dépôt et à la diffusion de documents scientifiques de niveau recherche, publiés ou non, émanant des établissements d'enseignement et de recherche français ou étrangers, des laboratoires publics ou privés. 


\title{
Numerical Study of the Influence of Flux Creep and of Thermal Effect on Dynamic Behaviour of Magnetic Levitation Systems with a High-Tc Superconductor Using Control Volume Method
}

Lotfi Alloui ${ }^{1}$, Frédéric Bouillault ${ }^{1}$, Souri Mohamed Mimoune ${ }^{2}$

${ }^{1}$ Laboratoire de Génie Electrique de Paris - LGEP; CNRS UMR 8507; Supelec; Univ Pierre et Marie Curie-P6; Univ Paris Sud-P11, Plateau de Moulon, 11 rue Joliot Curie, 91192 GIF-SUR-YVETTE Cedex, France

${ }^{2}$ Laboratoire de Modélisation des systèmes Energétique - LMSE; Département d'Electrotechnique, Université Mohamed Kheider, 07000 Biskra, Algeria

Email: lotfi.alloui@lgep.supelec.fr

\begin{abstract}
This paper displays some simulation results of dynamic responses of the high-Tc superconductors (HTSC)Permanent magnet (PM) levitation systems taking into account the influence of the flux creep phenomena and of the thermal effect. We focus on the establishment of a three-dimensional numerical code to solve the nonlinear and coupled equations. A new control volume method is proposed for the resolution of the partial derivative equations of the treaded physical phenomena. The influence is comprehensively displayed by comparing the predictions of dynamic responses of such systems in which the thermal effect in the superconductor is and is not taken into account. The electromagnetic and thermal coupling is ensured by an alternate algorithm. The thermal effect highlights the influence of the temperature on the value of the magnetic levitation force, levitation stabilization time and shows that the vibration center of levitated body had drifted downward.
\end{abstract}

PACS. 47.11.Df Finite volume methods - 84.71.Ba Superconducting magnets; magnetic levitation devices. 


\section{Introduction}

When the stable levitation phenomenon of the permanent magnet (PM) over the high temperature superconductor (HTSC) was found, several projects began to explore the potentials of the HTSC applications such as magnetic bearings [1], flywheel energy storage systems [2] and linear transportation system [3]-[4], etc.

The magnetic levitation transportation systems have the advantage of contactless support which offers high speed with low maintenance and few energy consumption. In the conception of these systems it becomes very important to evaluate precisely the electromagnetic force. Generally, these electromagnetic forces are influenced by the heat losses caused by induced current inside the superconductor. In this paper, the results show that heat losses lead to some important advantages and disadvantages.

In the levitation phenomena, the stability is due to the diamagnetic response of the High Tc superconductors and is greatly enhanced by the additional flux pining phenomenon: the magnetic field penetrates the HTSC and creates fluxoid (shielding current density). The magnetic force results from the interaction between the magnetic field and the fluxoid. The motion of these fluxoid leads to flux flow and flux creep phenomena that make the magnetic force time-dependent since the flux influences the dynamic magnetic force's characteristics of the superconductor.

Flux creep is observed both in the conventional low Tc superconductors and in the high Tc oxides. But flux creep is mainly present in high superconductors since usually high temperatures and low pinning potentials are involved. This is demonstrated, for instance, by the 'giant' flux creep and by the broadening of the resistive transition in magnetic fields which is a direct consequence of thermally activated flux creep.

Generally, the flux creep becomes an important phenomenon to consider. Yoshida et al. [5] who measured the dynamic magnetic force of HTSC show that good agreement between numerical results and measurement data are obtained only if flux creep is considered. In the literature, some macroscopic mathematical models were proposed to reflect the flux creep and the flux flow phenomena of superconductivity such as the model proposed by Anderson-Kim [6] or the model of power law proposed by Rhyner [7]. These models lead to good agreements with experimental data [9].

To represent the physical phenomena of the magnetic levitation, several works with different numerical methods and different magnetic formulations have been used to evaluate precisely the magnetic force which is the important parameter to consider [5]-[9]. However, these works do not take into consideration the thermal effect which can play an important role. The induced current in the superconductor creates a power density leading to heat transfer appearance and thus to a temperature increase. In practice, the temperature influences the dynamic magnetic force's characteristics of the superconductor. For 
instance, T. Suzuki and al [8]-[10], studied experimentally the effects of the temperature on the levitation force and the time relaxation. They found that the levitation force and the time relaxation of the levitation force decreased with increasing temperature. So it is interesting to develop a numerical code which allows dynamics behaviour modeling of magnetic levitation systems to take into account the thermal effect and which can explain the observed phenomena.

So, this paper presents 3D simulation results of dynamic responses of the high-Tc superconductor-PM levitation systems. After the description of the considered system, the mathematical models based on the A-V formulation and the numerical code which uses control volume method CVM for solving the nonlinear and coupled problem are examined.

\section{Basic formulation}

In this section we introduce the main equations of the superconductor HTSC - permanent magnet PM levitation system. In such system, a cylindrical PM moves (vertically or laterally) over a rectangular high Tc superconductor as shown in Fig.1.

The effect of flux creep in the superconductor is simulated by using a logarithmic dependence between the critical current and the thermodynamic activation energy, together with the Arrhenius law, as suggested in [11]. The resulting relation between the electrical field and the current density inside the superconductor can be written in the form of power law as:

$$
\mathbf{E}(\mathbf{J}, T)=E_{c}\left|\frac{\mathbf{J}}{J_{c}(T)}\right|^{n(T)} \frac{\mathbf{J}}{J}
$$

$J C$ is the critical current density which may depend of temperature $T$ and $E c$ is the critical field of the superconductor. On the basis of Anderson's excitation creep model, the exponent $n$ can be identified with $U c /\left(k_{B} T\right)$, where $U c$ is the critical activation energy and $k_{B}$ is Boltzmann's constant [12]. The limits cases of $n$ are the ohmic conductors law for ( $\left.n=1\right)$ and the Bean's model for $(n \rightarrow \infty)$. Generally, n varies from 5 to 25 to describe the flux creep behaviour [9]. For the used material in our work (YBaCuo), the good value of $\mathrm{n}$ is taken equal to 20 at $\mathrm{T}=77 \mathrm{~K}[13]$. In this paper we have adopted a linear model to describe the dependence of the critical current on temperature [19]:

$$
\begin{aligned}
& J_{c}(T)=J_{c 0} \frac{1-\frac{T}{T c}}{1-\frac{T_{0}}{T c}} \\
& n(T)=\frac{n_{0} T_{0}}{T} \text { with } n_{0}=\frac{U_{c}}{k_{B} T_{0}}
\end{aligned}
$$


$n_{0}, J_{c 0}$ are respectively the $n$-exponent and the critical current density at $T=T_{0}$ ( $\mathrm{T}_{0}$ is the liquid nitrogen bath temperature).

In addition to the gravity force, the PM is submitted to an electromagnetic force resulting from its interaction with the superconductor. This force is the opposite of the magnetic one exerted by the PM on the superconductor, ie:

$$
\mathbf{F}=\int_{\Omega} \mathbf{J} \times\left(\nabla \times \mathbf{A}_{\mathbf{s}}\right) d \Omega
$$

$\mathbf{J}$ is the shielding current density, $\Omega$ represents the volume of the superconductors and $\mathbf{A}_{\mathbf{s}}=\mathbf{A}_{\mathbf{m}}+\mathbf{A}$ stands for the total vector potential. A is the induced part of $\mathbf{A}_{\mathbf{s}}$ due the shielding current in the superconductor, and $\mathbf{A}_{\mathbf{m}}$ is the excitation part of $\mathbf{A}_{\mathbf{s}}$ due to the presence of PM. For the calculation of $\mathbf{A}_{\mathbf{m}}$, a permanent magnet with a magnetic moment $\mathbf{m}$ has been modelled by succession of loops with surface current density $\mathbf{j}=\mathbf{m} \wedge \mathbf{n}[14]$. By using the Biot-Savart law, $\mathbf{A}_{\mathbf{m}}$ is calculated in each point of the whole domain.

The A-V formulation (V: is the electric scalar potential) is used to calculate the induced parts of $\mathbf{A}_{\mathbf{s}}$ given by:

$$
\begin{aligned}
& \nabla \times\left(v_{0} \nabla \times \mathbf{A}\right)+\sigma(\mathbf{E}, T)\left(\frac{\partial \mathbf{A}}{\partial t}+\nabla V\right)=\mathbf{J}_{\mathbf{s}} \\
& \nabla\left\{-\sigma(\mathbf{E}, T)\left(\frac{\partial \mathbf{A}}{\partial t}+\nabla V\right)\right\}=0
\end{aligned}
$$

Where $v_{0}$ is the magnetic reluctivity of vacuum used for superconducting and nonconducting parts, and $\mathbf{J}_{\mathbf{s}}$ is the source current density defined by:

$$
\mathbf{J}_{\mathbf{s}}=-\sigma(\mathbf{E}, T) \frac{\partial \mathbf{A}_{\mathbf{m}}}{\partial t}
$$

In (4) the magnetic vector potential $\mathbf{A}$ can not be unique unless a gauge constraint is used. The most useful one is the Coulomb gauge $(\nabla . \mathbf{A}=0)$ introduced in $(4)$ by adding a penalty term [15]-[16]. Then the left hand size of the first equation in (4) becomes a Laplacien: 


$$
\begin{aligned}
& \nabla \times\left(v_{0} \nabla \times \mathbf{A}\right)-\nabla v_{0} \nabla \cdot \mathbf{A}+\sigma(\mathbf{E}, T)\left(\frac{\partial \mathbf{A}}{\partial t}+\nabla V\right)=\mathbf{J}_{\mathbf{s}} \\
& \nabla \cdot\left\{-\sigma(\mathbf{E}, T)\left(\frac{\partial \mathbf{A}}{\partial t}+\nabla V\right)\right\}=\mathbf{0}
\end{aligned}
$$

$\sigma$ is the nonlinear equivalent conductivity of the superconductor and is defined using (1) by:

$$
\sigma(\mathbf{E}, T)=\frac{J c(T)}{E c}\left(\frac{|\mathbf{E}|}{E c}\right)^{\frac{1}{n(T)}-1}
$$

So in the superconducting region it is necessary to evaluate

$$
\mathbf{E}=-\frac{\partial \mathbf{A}_{\mathbf{m}}}{\partial t}-\frac{\partial \mathbf{A}}{\partial t}-\nabla V
$$

In order to obtain the distribution of the temperature in the superconductor, we should resolve the thermal equation in the superconductor:

$$
\rho(T) C_{p}(T) \frac{\partial T}{\partial t}-\nabla \cdot(\kappa(T) \nabla T)=W
$$

where $\kappa(T)$ is the thermal conductivity, $\rho(T) C p(T)$ is the heat capacity per volume, and $W=\sigma(\mathbf{E}, T) . \mathbf{E}^{2}$ is the power magnetic density generated by the electromagnetic phenomenon in the superconductor's sample. Convective boundary conditions are used:

$$
-\kappa(T) \nabla T \cdot \mathbf{n}=h\left(T-T_{o}\right)
$$

which expresses the thermal flux exchange between the superconductor and the cryogenic fluid. In (10), $h$ is the convective heat transfer coefficient. 
The superconducting material used in this application is the $\mathrm{YBaCuO}$. The thermal and electrical characteristics of $\mathrm{YBaCuO}$ are given in table I extracted from literature [13-17-18-19].

To determine the position of the PM, we have to study the movement of the levitated body in vertical (z) and lateral (x) directions. Using the second Newton's law, the equation of movement of the PM when the air damping is neglected, can be expressed as:

$$
\sum \mathbf{F}=m \gamma \Rightarrow\left\{\begin{array}{l}
F_{z}-m g=m \ddot{z} \\
F_{x}=m \ddot{x} \\
F_{y}=m \ddot{y}
\end{array}\right.
$$

Taking into account initial conditions:

$$
t=0:(x, y, z)=\left(x_{0}, y_{0}, z_{0}\right) \text { and }(\dot{x}, \dot{y}, \dot{z})=\left(\dot{x}_{0}, \dot{y}_{0}, \dot{z}_{0}\right)
$$

Here $F_{z}, F_{x}$ and $F_{y}$ are the component of the electromagnetic force $\mathbf{F}$ given by equation (3), $m$ is the mass of the permanent magnet and $g$ is the gravitational acceleration. Using equation (11), it's possible to determine the new position of PM due to the variation of the field.

\section{Control Volume Method}

The numerical method used in this paper is the control volume method (CVM). This method can be regarded as a special version of the weighted residuals method. It consists in dividing the domain into a number of nonoverlapping subdomains or control volumes such that there is one control volume surrounding each node (Fig.2.a). The weighting function is setting to be unity over one subdomain at a time and zero everywhere else. This variant of the method of weighted residuals is called the subdomain method or CVM.

The basic formulation of CVM deals with differential equations which seem to obey a conservation principle defined by the divergence law. In this case, six nodes surrounding each principle node ' $\mathrm{P}$ ' are necessary to discretize the algebraic equation : West 'W', East 'E', South 'S', North 'N', bottom 'B' and Top 'T' (Fig.2.a). This basic formulation with seven nodes in total is suitable for heat and mass transfer, fluid flow, chemical reaction and others related processes. 
In the general case, where the magnetic permeability takes different values, this basic formulation is not well appropriated for the electromagnetic equation (4) which obeys a conservation principle defined by the rotational law. Then it is necessary to modify this basic formulation to derive the algebraic equations. In order to do this, twelve additional nodes are needed WS, $\mathrm{WN}, \mathrm{ES}, \mathrm{EN}, \mathrm{SB}, \mathrm{ST}, \mathrm{NB}, \mathrm{NT}, \mathrm{BW}, \mathrm{BE}, \mathrm{TW}$ and $\mathrm{TE}$, so in total twenty one nodes are required. Figure (2.b) presents a projection of a new control volume according to plan X-Y.

The system of equations in (6) will be integrated over the control volume $v^{e}$ as follow

$$
\begin{aligned}
& \iiint_{\mathrm{v}^{e}} \nabla \times(v \cdot \nabla \times \mathbf{A}) d x d y d z-\iiint_{\mathrm{v}^{e}} \nabla\left(\mathrm{v}_{0} \cdot \nabla \cdot \mathbf{A}\right) d x d y d z+\iiint_{\mathrm{v}^{e}} \sigma\left(\frac{\partial \mathbf{A}}{\partial t}+\nabla V\right) d x d y d z=\iiint_{\mathrm{v}^{e}} \mathbf{J}_{\mathbf{s}} d x d y d z \\
& \iiint_{\mathrm{v}^{e}} \nabla \cdot\left\{-\sigma\left(\frac{\partial \mathbf{A}}{\partial t}+\nabla V\right)\right\} d x d y d z=0
\end{aligned}
$$

System (13) gives differential terms of second order. The terms of the two identical first order derivative lead to the classical basic control volume scheme which handles with free divergence laws (figure 2a), for example:

$$
\int_{w}^{e} \int_{s}^{n} \int_{b}^{t}\left[\frac{\partial}{\partial x} v\left(\frac{\partial \mathbf{A}_{y}}{\partial x}\right)\right] d x d y d z=\left.\left\{v\left(\frac{\partial \mathbf{A}_{\mathbf{y}}}{\partial x}\right)\right\}\right|_{w} ^{e} \Delta y \Delta z
$$

An approximation of first order is used to evaluate numerically expression (14):

$$
\left.\left(v \frac{\partial \mathbf{A}_{\mathrm{y}}}{\partial x}\right)\right|_{w} ^{e}=\left(\left.v_{e} \frac{\partial \mathbf{A}_{\mathrm{y}}}{\partial x}\right|_{e}-\left.v_{w} \frac{\partial \mathbf{A}_{\mathrm{y}}}{\partial x}\right|_{w}\right)=\left(v_{e} \frac{\mathbf{A}_{y}^{\mathrm{E}}-\mathbf{A}_{y}^{\mathrm{P}}}{\Delta x_{e}}-v_{w} \frac{\mathbf{A}_{y}^{\mathrm{P}}-\mathbf{A}_{y}^{\mathrm{W}}}{\Delta x_{w}}\right)
$$

After arranging, (15) leads to the algebraic relationship written as:

$$
c_{e} \mathbf{A}_{\mathrm{y}}^{\mathrm{E}}+c_{w} \mathbf{A}_{\mathrm{y}}^{\mathrm{W}}-\left(c_{e}+c_{w}\right) \mathbf{A}_{\mathrm{y}}^{\mathrm{P}}
$$

The terms of the second order crossed derivatives lead to the new control volume scheme (figure 2b), for example: 


$$
\int_{w}^{e} \int_{s}^{n} \int_{b}^{t}\left[\frac{\partial}{\partial y} v\left(\frac{\partial \mathbf{A}_{\mathrm{y}}}{\partial x}\right)\right] d x d y d z=\left.\left\{v\left(\frac{\partial \mathbf{A}_{\mathrm{y}}}{\partial x}\right)\right\}\right|_{s} ^{n} \Delta x \Delta z
$$

Using this new configuration of the control volume, the magnetic potential in the equation (14) will be calculated in the following way:

$$
\begin{aligned}
& \left.\left\{v\left(\frac{\partial \mathbf{A}_{\mathbf{y}}}{\partial x}\right)\right\}\right|_{\mathrm{s}} ^{\mathrm{n}}=v_{n} \frac{\mathbf{A}_{\mathrm{y}}^{\mathrm{ne}}-\mathbf{A}_{\mathrm{y}}^{\mathrm{nw}}}{\Delta x}-v_{s} \frac{\mathbf{A}_{\mathrm{y}}^{\mathrm{se}}-\mathbf{A}_{\mathrm{y}}^{\mathrm{sw}}}{\Delta x}= \\
& \frac{v_{n}}{4 \Delta x}\left(\mathbf{A}_{\mathrm{y}}^{\mathrm{NE}}+\mathbf{A}_{\mathrm{y}}^{\mathrm{E}}-\mathbf{A}_{\mathrm{y}}^{\mathrm{NW}}-\mathbf{A}_{\mathrm{y}}^{\mathrm{W}}\right)-\frac{v_{s}}{4 \Delta x}\left(\mathbf{A}_{\mathrm{y}}^{\mathrm{SE}}+\mathbf{A}_{\mathrm{y}}^{\mathrm{E}}-\mathbf{A}_{\mathrm{y}}^{\mathrm{SW}}-\mathbf{A}_{\mathrm{y}}^{\mathrm{W}}\right)
\end{aligned}
$$

After arranging, (18) leads to an algebraic relationship written as:

$$
c_{y n}\left(\mathbf{A}_{\mathrm{y}}^{\mathrm{NE}}+\mathbf{A}_{\mathrm{y}}^{\mathrm{E}}-\mathbf{A}_{\mathrm{y}}^{\mathrm{NW}}-\mathbf{A}_{\mathrm{y}}^{\mathrm{W}}\right)-c_{y s}\left(\mathbf{A}_{\mathrm{y}}^{\mathrm{SE}}+\mathbf{A}_{\mathrm{y}}^{\mathrm{E}}-\mathbf{A}_{\mathrm{y}}^{\mathrm{SW}}-\mathbf{A}_{\mathrm{y}}^{\mathrm{W}}\right)
$$

All integrant terms resulting from system (13) are evaluated. Therefore, all the coefficients $c_{i}$ are obtained and describe the physical and the geometrical properties of the cellule surrounding each node. An algebraic system of equations is constructed with appropriate boundaries conditions in the whole domain and expressed as:

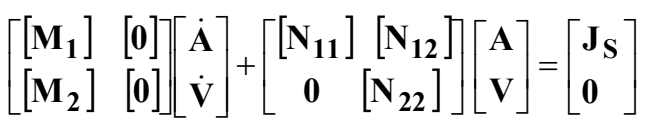

where the column matrix $\left[\begin{array}{c}\mathbf{A} \\ \mathbf{V}\end{array}\right]$ denotes nodal values of the magnetic vector potential and the electrical scalar potential; $\left[\begin{array}{c}\dot{\mathbf{A}} \\ \dot{\mathbf{V}}\end{array}\right]$ means the column matrix of differentiation of $\mathrm{A}$ and $\mathrm{V}$ with respect to time variable and $\left[\begin{array}{l}\mathbf{J}_{\mathbf{S}} \\ \mathbf{0}\end{array}\right]$ represents the column matrix of the permanent magnet excitation related to the last term of (6). 
The global control volume matrices $[\mathbf{M}]$ and $[\mathbf{N}]$ are non symmetric and non linear. According to the definition of conductivity given by (7), we know that the effective conductivity $\sigma$ of the superconductor is nonlinearly dependent on both electric field intensity $\mathbf{E}$ and shielding current density $\mathbf{J}$. At each step, the Crank-Nicolson method is employed to integrate the algebraic system (20) which is solved iteratively until convergence is reached.

In the same way the control volume method is used to solve the algebraic equation of the differential thermal equation (9). The obtained algebraic system when taking into account the boundary conditions (10) is

$$
[\mathbf{C}][\mathbf{T}]+[\mathbf{K}][\mathbf{T}]=[\mathbf{Q}]
$$

Here, $[\mathbf{T}]$ denotes the column matrix of the nodal values of the temperature; $[\dot{\mathbf{T}}]$ the column matrix of differentiation of $T$ with respect to time variable and $[\mathbf{C}]$ is a nonlinear matrix related to the column matrix $[\dot{\mathbf{T}}]$ and $[\mathbf{K}]$ the linear matrix related to $[\mathbf{T}]$. [Q] represents the column matrix related to power density and heat exchange in the superconductor. The system is also solved iteratively using the Crank-Nicolson method to treat the time derivative term.

The coupling algorithm of the electromagnetic and thermal equations consists of an iterative algorithm based on the bloc diagonal Gauss-Seidel method. It permits to separate the non symmetric and the non linear magnetic equation from the non symmetric and non linear thermal one.

\section{Numerical results and discussion}

The developed numerical code is used to display some tests results of considered levitation systems. On the one hand, the first use of the numerical code allows simulating the behaviour of the sample superconductor without taking into account the thermal effect. On the other hand it permits to validate the code. The goals of the simulation are to obtain a qualitative physical understanding of the stability features and to describe the difference between cooling the superconductor close to the magnet and cooling it at long distance. We can also predict the appearance of lateral restoring forces and calculate what happens when arbitrary displacements of the magnet occur around the superconductor. The levitated system consists of a rectangular-shaped superconductor (36 mm long, $36 \mathrm{~mm}$ wide and $10 \mathrm{~mm}$ thick) and a cylindrical PM (20 mm diameter, $10 \mathrm{~mm}$ thick and $250 \mathrm{~g}$ weight).

Fig.3(a,b,c) shows the magnetic induction vector field and the current density distribution of the component $J_{y}$ which arise when the permanent magnet polarized of $1.1 \mathrm{~T}$ goes down toward a cool superconductor. The starting position is located 
at $20 \mathrm{~mm}$ from the superconductor, then the PM will be brought closer to the superconductor at a constant speed rate and will be stopped at $2 \mathrm{~mm}$ (Fig.3.c). We observe that the magnetic field due to PM penetrates the HTS bulk from the upper side, and is excluded in the lower part of the sample. The variation in time of this field induces a current density in the region penetrated by the field and leads to a repulsion force between the PM and the superconductor (see fig.3.d). Therefore, we conclude that is not necessary to use a superconductor with large height, indeed the superconductor develops a surface current such as the current penetration depth is sufficient to exclude the field created by the magnet. Over this depth, the contribution of the bottom tends progressively to be negligible.

The levitation force in Fig.3.d, which is calculated when the superconductor is cooling at long distance to the magnet, shows a hysteresis property. A repulsive force is obtained when the permanent magnet is moved toward the HTSC as shown in upper line in Fig. 3.d. If the magnet is next moved away from the HTSC, the repulsive force decreases and a weak attractive force appears, which is called the fishing effect [12]-[20].

Fig.4(a,b,c) shows the magnetic induction vector field and the current density distribution of the component $J_{y}$ when the PM is first close to the surface of the superconductor, then moves up from the superconductor and finally goes down. In this case a new physical phenomenon arises. In the first step when the PM is close to the surface of the superconductor (Fig.4.a) this later is cooled. In this case, the superconductor stays passive and does not develop induced current. This can be explained by the fact that the flux structure due to the magnet is frozen within the sample and the condition $\mathbf{J s}=\nabla \times \mathbf{H}=\mathbf{0}$ is fulfilled. Thus no change occurs until the magnet is shifted. In the second step when the magnet is moved away from the superconductor, a current distribution appears due to the variation in the time of the magnetic field (Fig.4.b). We can notice that in this case, this current induces an attractive force (see Fig.4.d). If the movement of the magnet is reversed, the interaction force becomes repulsive because the new induced current flows in the opposite direction to the previous scheme (see Fig.4.c), there is no hysteresis property. Fig.4.d shows the actual behaviour of the levitation force for a specific process.

Fig.5.(a,b,c) shows the magnetic induction vector field and the current density distribution of the component $J_{y}$ that appear when the magnet is laterally displaced. Notice that these studies can be obtained only by using a 3D approach contrary to the previous ones where 2D modeling can lead to approximated results. An attractive X horizontal force appears when the PM is shifted to the right hand side of the superconductor ( $\mathrm{x}$ direction). This force increases until the edge of the PM becomes close to the edge of the superconductor, after that the force decreases and tends to zero (Fig.5.d).

The numerical code is examined a second time in order to predict the experimental phenomena which arise when the temperature is increased and thus the force is reduced [8]-[10]. This effect can be observed for example in the dynamic 
response of the levitation behaviour [8]-[10]. First, some basic simulations are made to show if the influence of the superconductor's temperature on the levitation behaviour is notable. The results of theses simulations point out the difference between the displacement responses and the magnetic force of the levitation system when the thermal effect is and is not considered. Fig.6 and fig.7 display the dynamic response of the displacement and the magnetic force of a free levitated PM, where the initial conditions are taken as $\mathrm{z}_{0}=8 \mathrm{~mm}$ and $\dot{z}_{0}=0$, respectively.

In fig.6, it is found that there is a noticeable downward drift of the vibration centre in the response. The thermal effect does not appear initially. But from the instant $t=0.22 \mathrm{~S}$ a shift appears between the dynamics obtained from the model which takes into account the thermal effect and the one which does not. We can conclude that the thermal effect can not be neglected. By comparing the dynamics of the levitation system obtained we can see that the thermal effect accelerates the stability of the levitation body.

Fig.7 displays the magnetic force varying with time. From it, one sees that when the thermal effect is taken into account, the magnetic force decays faster than when the thermal effect is not considered. This can be explained by the fact that the thermal effect in the high Tc superconductor due to an increasing temperature leads to a decreasing current density.

Figure 8 shows the evolution of the temperature as a function of time along $Z$ axis including the center of the superconductor. Figure 9 shows the temperature profile in superconductor at different instants $\mathrm{t}=0.5 \mathrm{~s}, \mathrm{t}=1$ and $\mathrm{t}=1.575 \mathrm{~s}$, respectively. From these figures, we can see that the temperature increases from the top (near to the power source) to the bottom of the superconductor.

Fig. 10 shows the dependence of the final stable position (static equilibrium position) of the levitation system with the initial position $z_{0}$. This result is very important in the levitation systems application. The value of the final stable position of the PM depends strongly on the $z_{0}$ value. The final stable position becomes higher when the initial position is elevated. Therefore the initial position $\mathrm{z}_{0}$ plays an important role in the levitated body.

Let $z_{\text {ths }}$ and $z_{s}$ be the final stable positions of the levitated PM when the thermal effect is and is not considered in the numerical simulation, respectively. Denote their absolute error by $\varepsilon=\left|\mathrm{z}_{\mathrm{ths}}-\mathrm{z}_{\mathrm{s}}\right|$. It is evident that the error $\varepsilon$ characterizes the influence of the thermal effect in the superconductors on the superconducting levitation systems. The prediction exhibits that the absolute error is mainly dependent on the initial position $z_{0}$ for a free levitated PM. In this case the characteristic curve of the absolute error varying with the initial position $z_{0}$ is plotted in figure 11 . From it, we see that the absolute error increases where the initial position $\mathrm{z}_{0}$ of the PM increases. When the initial position is fewer than $4 \mathrm{~mm}$ the absolute error becomes small and the influence of the thermal effect can be neglected. 


\section{Conclusions}

In this paper, the shielding current and the electromagnetic field in the superconductor are determined by solving the A-V magnetic equation coupled to the thermal one in order to show the influence of the temperature on the dynamic behaviour of the levitation systems. The flux creep phenomenon is described by using the power law relationship. A three-dimensional numerical code is employed to simulate the dynamic responses of the high-Tc superconductor-PM levitation system. The code is based on a new control volume method for the spatial scheme and a Cranck-Nickolson algorithm for the time one. An iterative solver is used for the resolution of the final algebraic coupled equations. The numerical results exhibit that the drift of the vibration center of the levitation systems is mainly dependent upon the thermal effect when the initial position of the PM above a superconductor is large enough. Hence, the influence of the thermal effect in the superconductor model which leads to larger drift of vibration center, has to be considered in the dynamic simulation of a high superconductor PM levitation system in general case. 


\section{References}

1. I. Masaie, K. Demachi, T. Ichihara, and M. Usaka, "Numerical evaluation of rotational speed degradation in the superconducting magnetic bearing for various superconducting bulk shapes,', IEEE Trans. Appl. Supercond., vol. 15, No.2, pp. 2257-2260, Jun. 2005.

2. J. Hull, “'Superconducting bearings,’’ Supercond. Sci. Technol., vol. 13, pp. R1-R15, 2000.

3. J. Wang and al., "The first man-loading high temperature superconducting maglev test vehicle in the world,' Physica C, vol. 378-381, pp. 809-814, 2002.

4. Z. Ren, J. Wang, S. Wang, H. Jiang, M. Zhu, X. Wang and X. Shen, “A hybrid maglev vehicle using permanent magnets and high temperature superconductor bulks,', Physica C, vol. 378-381, pp. 873-876, 2002.

5. Y. Yoshida, M. Uesaka, and K. Miya, "Magnetic field and force analysis of high Tc superconductor with flux flow and creep,' IEEE Trans. Magn, vol. 30, no. 5, pp. 3503-3506, Sep. 1994.

6. P. W. Anderson, “Theory of flux creep in hard superconductors,” Phys. Rev. Lett., vol. 9, pp. 309-311, 1962.

7. J. Rhyner, "Magnetic properties and ac losses of superconductors with power law current-voltage characteristics," Physica C, vol. 212, pp. 292-300, 1993.

8. H. Jiang, J. Wang, S. Wang, Z. Ren, M. Zhu, X. Wang, and X. Shen, “The magnetic levitation performance of YBaCuO bulk at different temperature,'’ Physica C, 378-381 (2002) pp. 869-872, February 2002.

9. R. B. Kasal, R. de Andrade, Jr., G. G. Sotelo, and A. C. Ferreira, “Simulation of dynamic levitation force taking flux creep into account”' IEEE Trans. Appl. Supercond., vol. 17, no.2, pp. 2158-2161, Jun. 2007.

10. T. Suzuki, E. Ito, T. Sakai, S. Koga, M. Murakami, K. Nagashima, Y. Miyazaki, H. Seino, N. Sakai, I. Hirabayashi, and K. Sawa, "Temperature dependency of levitation force and its relaxation in HTS', IEEE Trans. Appl. Supercond., vol. 17, no.2, pp. 3020-3023, Jun. 2007. 
11. E. H. Brandt," Superconductor disks and cylinders in an axial magnetic field. I. Flux penetration and magnetization curves,' Physical Review B, vol.58, no. 10, pp. 6506-6522, September 1998.

12. T. Sugiura, H. Hashizume and K. Miya, “Numerical electromagnetic field analysis of type-II superconductors,' Int.J. Appl. Electromagn. in Mat., vol. 2, pp183-196, 1991.

13. K. Berger, “Etude des phénomènes couples magnétothermiques dans les supraconducteurs à haute température,’ Thése de l’université Henri Poincaré, Nancy I, septembre 2006, France.

14. S. H. T. Fou, A. Erraud, and F. Bouillault, "Numerical Modelling of the association of magnet and HTS superconductors,' IEEE Trans. Magn, vol. 36, no. 4, pp. 1197-1200, July. 2000.

15. J. L. Coulomb, “Finite element three dimensional magnetic field computation,’ IEEE Trans. Magn, vol. 17, pp. 32413246, Nov. 1981.

16. O. Biro and K. Preis, "On the use of the magnetic vector potential in the finite element analysis of $3 \mathrm{~d}$ eddy currents," IEEE Trans. Magn, vol. 25, pp. 3145-3159, July. 1989.

17. Comment on condensed matter physics, vol14, $\mathrm{N}^{\circ} 2, \mathrm{p} 65,1988$.

18. M. Lkebe and al,“Anisotropic thermal diffusivity and conductivity of $\mathrm{YBCO}(123)$ and $\mathrm{YBCO}(211)$ mixed crystals,” Jpn. J.appl. Phys. Vol.33(1994), p4965 and 6157.

19. S. Braeck, D. V. Shantsev, T. Johansen and Y. M. Galperin, "Superconducting trapped-field magnets: temperature and field distributions during pulsed-field activation,' Journal of applied physics, vol 92, pp. 6235-6240, 2002.

20. M. Murakami, “'Melt processed high-temperature superconductors,'’ World Scientific Publ. Co., 1992. 


\section{Figure captions}

Fig. 1. A high Tc-superconducting-PM levitation system

Fig. 2. a) Basic control volume scheme, b) New control volume scheme

Fig. 3. Current density distribution of the component $J_{y}$ in an HTSC puck and magnetic field induction of an HTSC-magnet system. The figures correspond to different gaps as the magnet is brought close to the HTSC and then brought away. The arrows indicate the direction of the movement of the magnet; a) gap $=20 \mathrm{~mm}$; b) gap $=2 \mathrm{~mm}$, c) gap $=20 \mathrm{~mm}$; d) forcedisplacement plot. Minimum gap $=2 \mathrm{~mm}$.

Fig.4. Current density distribution of the component $J_{y}$ in an HTSC puck and magnetic field induction of an HTSC-magnet system. The figures correspond to different gaps as the magnet is brought away to the HTSC and then brought close. The arrows indicate the direction of the movement of the magnet; a) gap = 2mm; b) gap = $21 \mathrm{~mm}$; ) gap $=4 \mathrm{~mm}$; d) forcedisplacement plot. A positive sign corresponds to repulsion, and a negative sign means attraction.

Fig.5. Current density distribution of the component $J_{y}$ in an HTSC puck and magnetic field induction of an HTSC-magnet system correspond to different lateral displacement. The arrows indicate the direction of the movement of the magnet. (a) lateral displacement $=2.1 \mathrm{~mm}$; (b) lateral displacement $=5.1 \mathrm{~mm}$, (c) lateral displacement $=7 \mathrm{~mm}$, and (d) represent horizontal force-displacement plot. A negative sign corresponds to attraction.

Fig.6. Simulation of response of the levitated PM of the levitation system when the thermal effect is and is not taken into account.

Fig.7. Simulation of magnetic force excreted on the levitated PM of the levitation system when the superconductor is dealt with and without thermal effect

Fig.8. Evolution of the superconductor's temperature.

Fig.9. Temperature distribution in superconductor at different instants respectively at $\mathrm{t}=0.25, \mathrm{t}=0.75 \mathrm{~s}$ and at $\mathrm{t}=1.47 \mathrm{~s}$.

Fig.10. the dependence of the final stable position (static- equilibrium position) of the freely levitated PM from the superconductor with the initial position

Fig.11. absolute error of prediction of response displacement between two cases of the superconductor with and without consideration the thermal effect 
$-16-$

Fig.1 Alloui et al EPJ Applid Physics

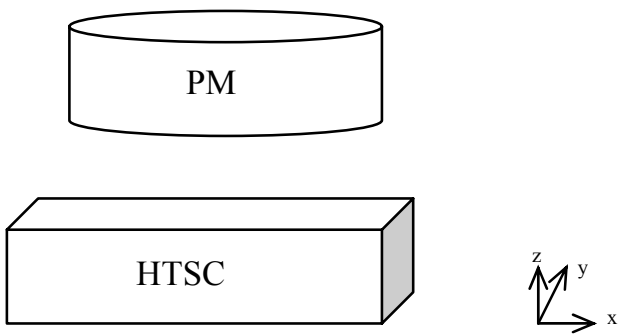


Fig. 2 Alloui et al EPJ Applied Physics

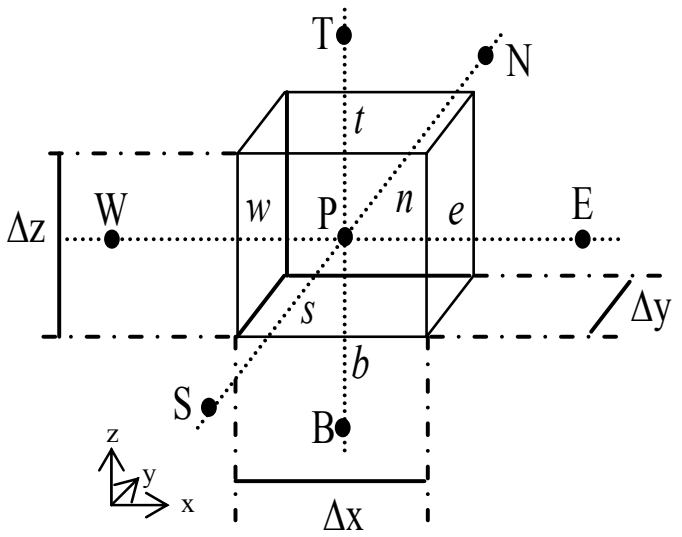

a)

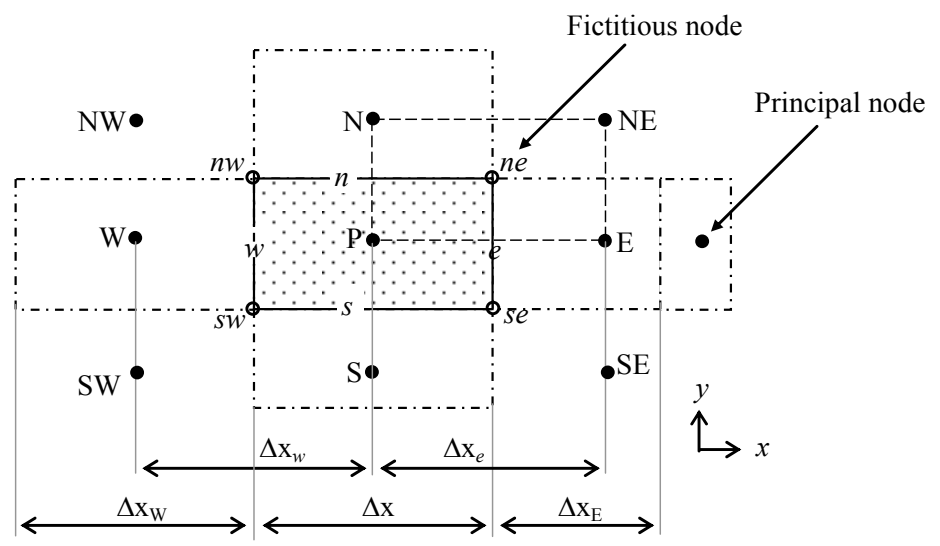

b) 
Fig. 3 EPJ Applied Physics

a)
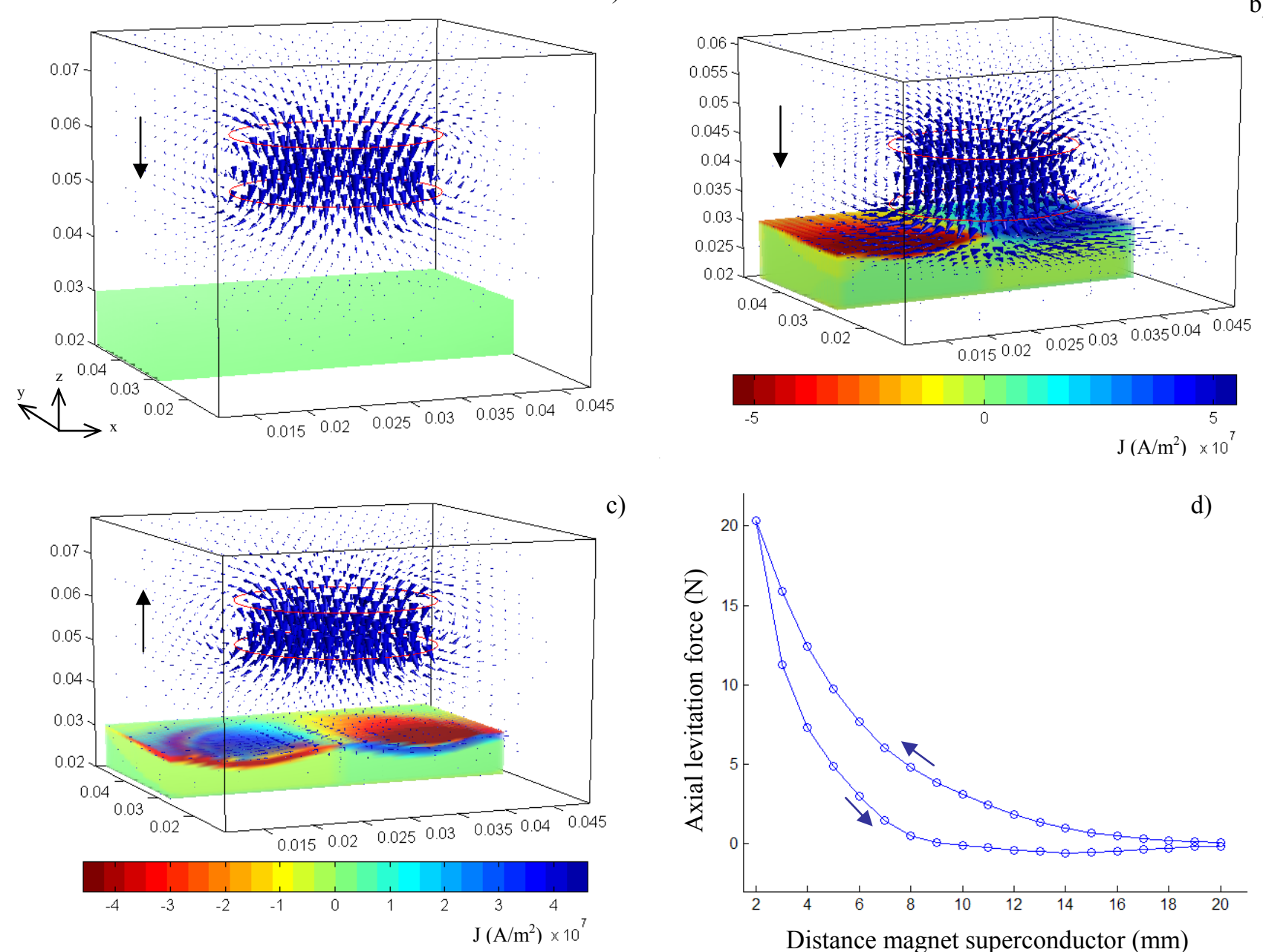

c)

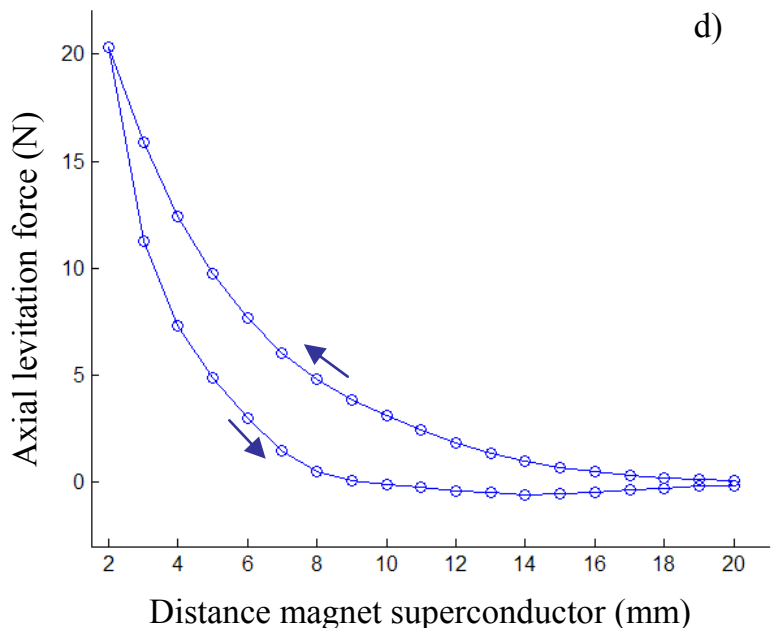

b)

d) 
Fig. 4

Alloui et al EPJ Applied Physics

a)

b)
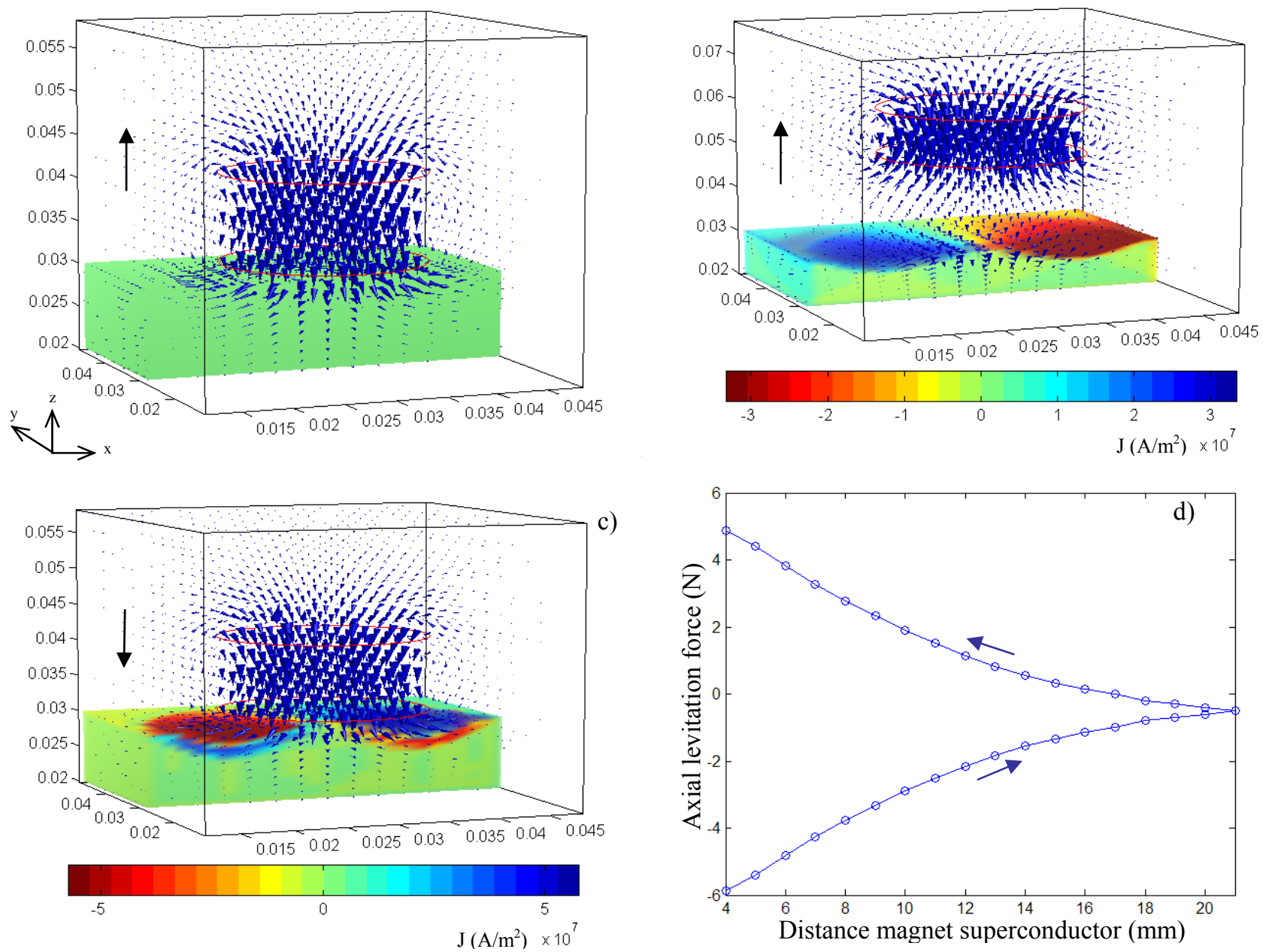
Fig. 5

Alloui et al EPJ Applied Physics
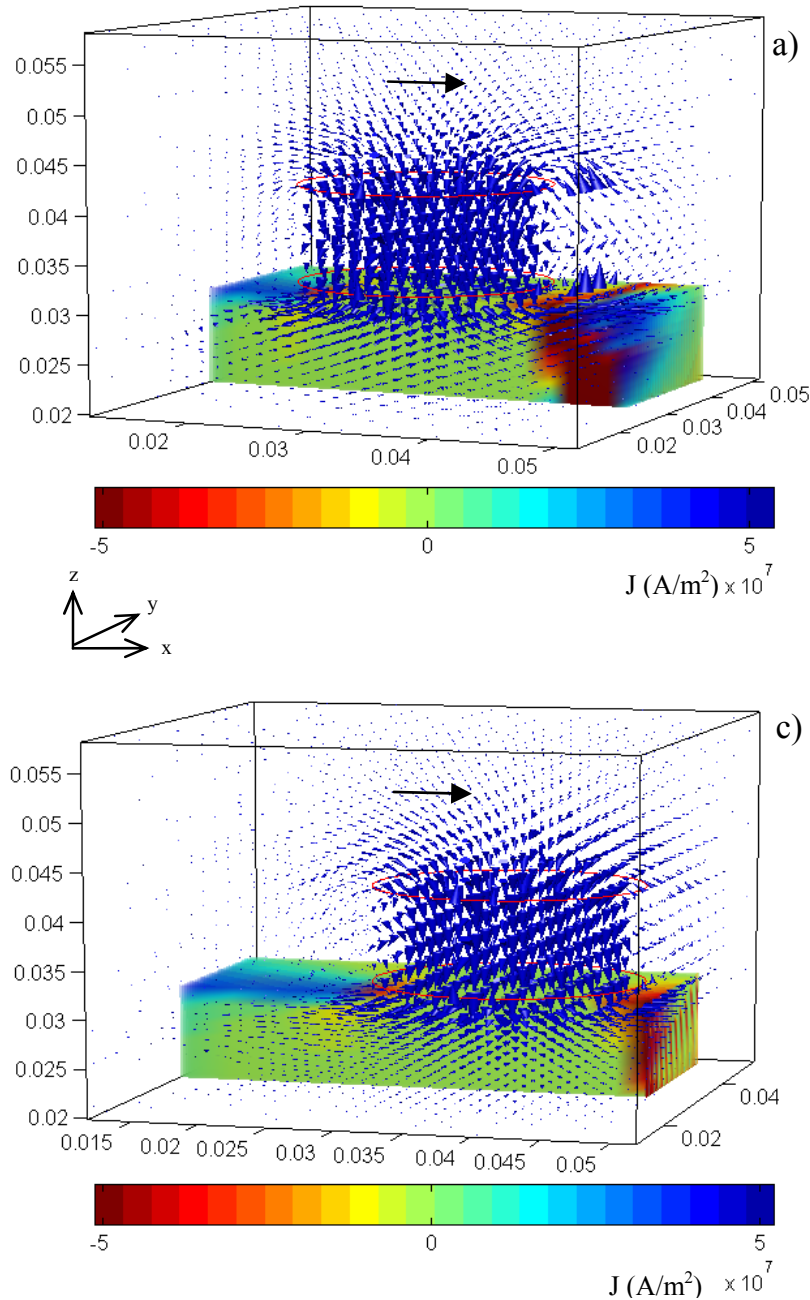

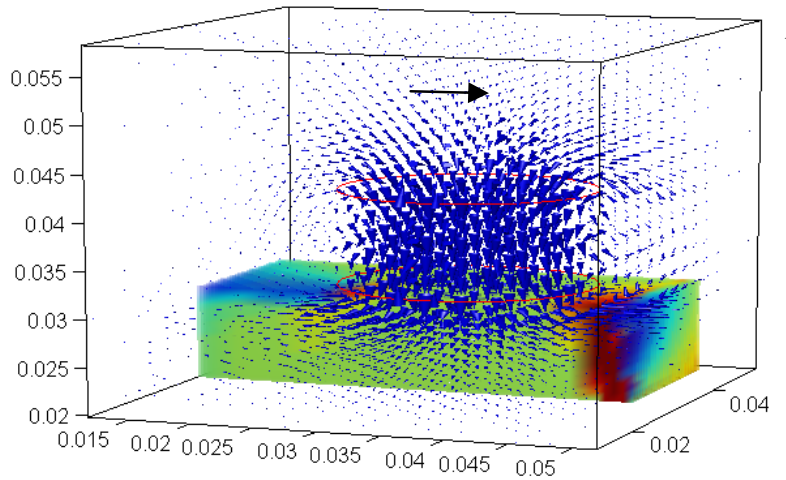

b)

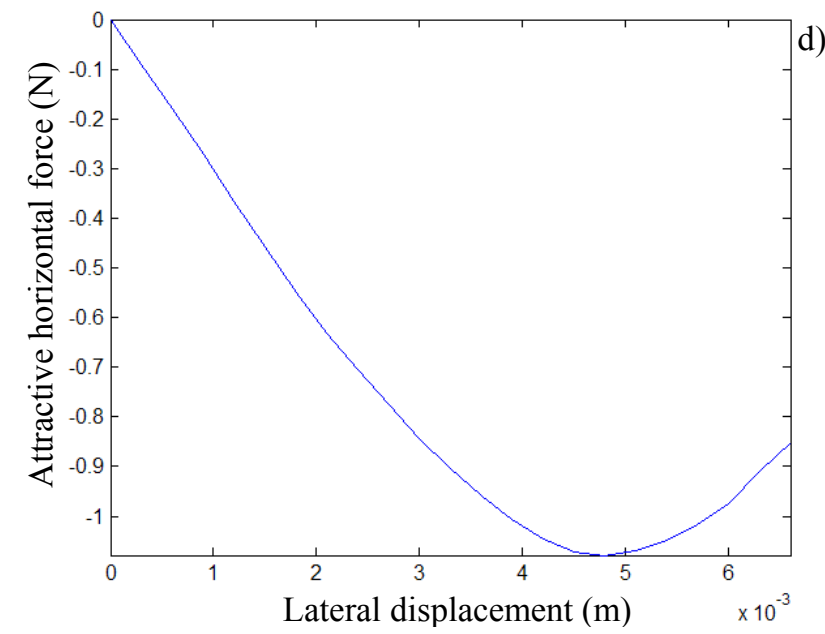




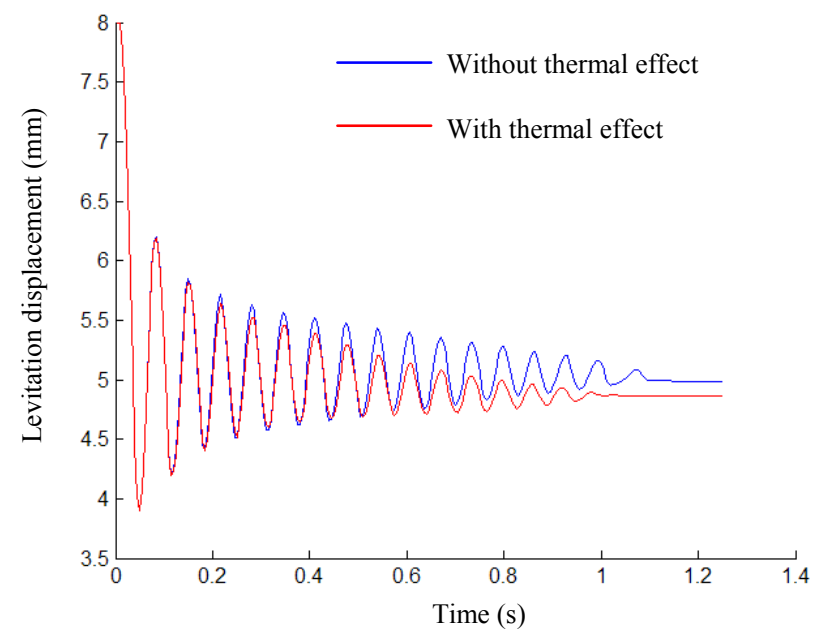


Fig. 7

Alloui et al EPJ Applied Physics

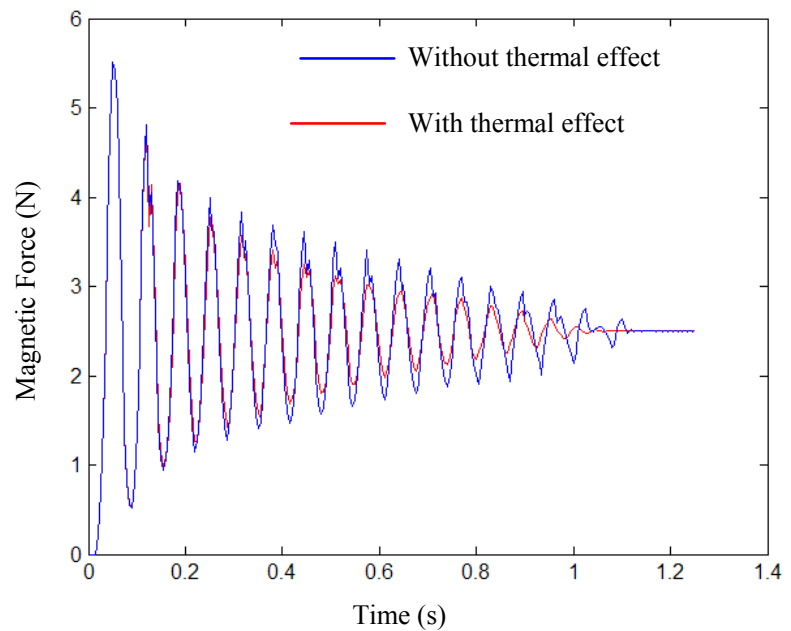


Fig. 8

Alloui et al EPJ Applied Physics

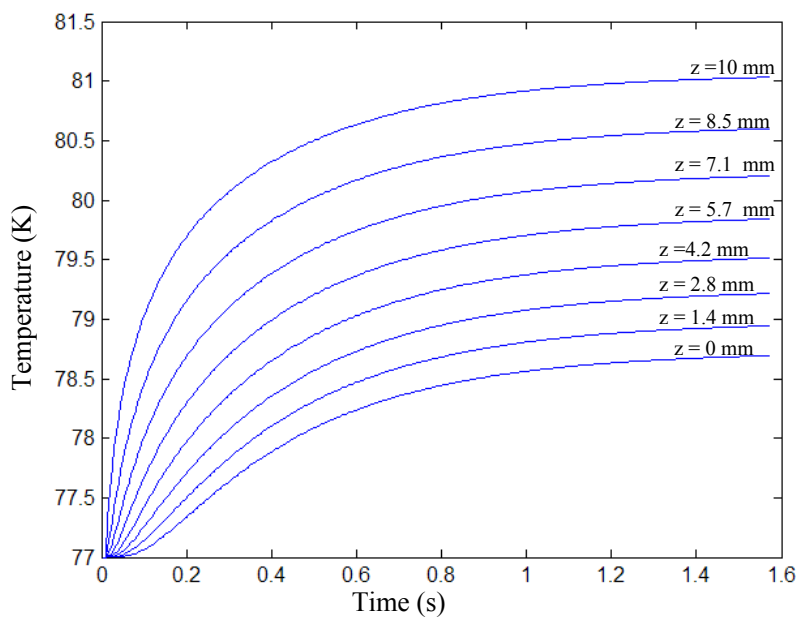


Fig. 9 Alloui et al
EPJ Applied Physics

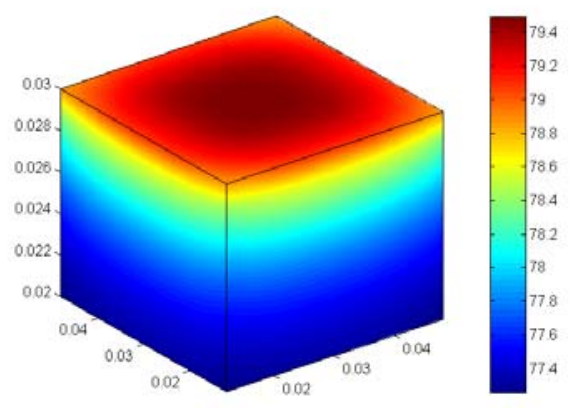

a)

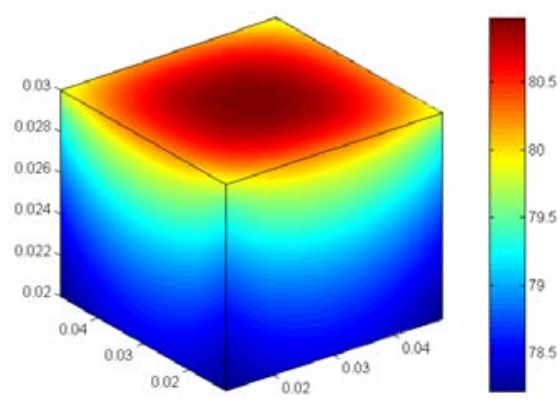

b)

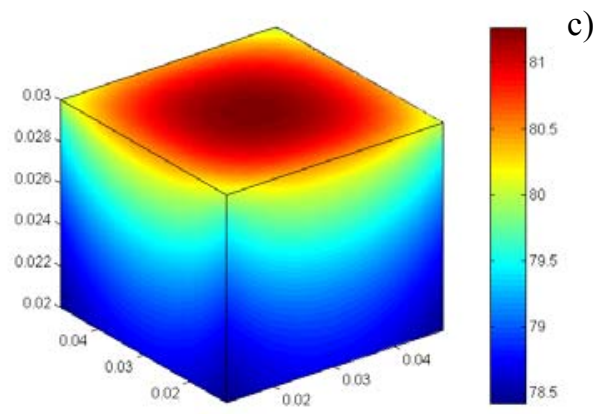


Fig. 10

Alloui et al EPJ Applied Physics

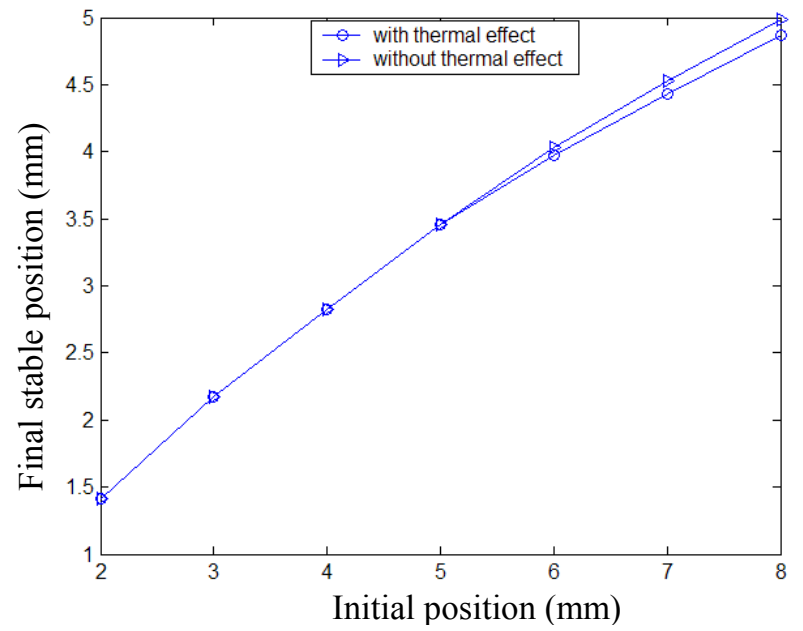


Fig. 11 Alloui et al EPJ Applied Physics

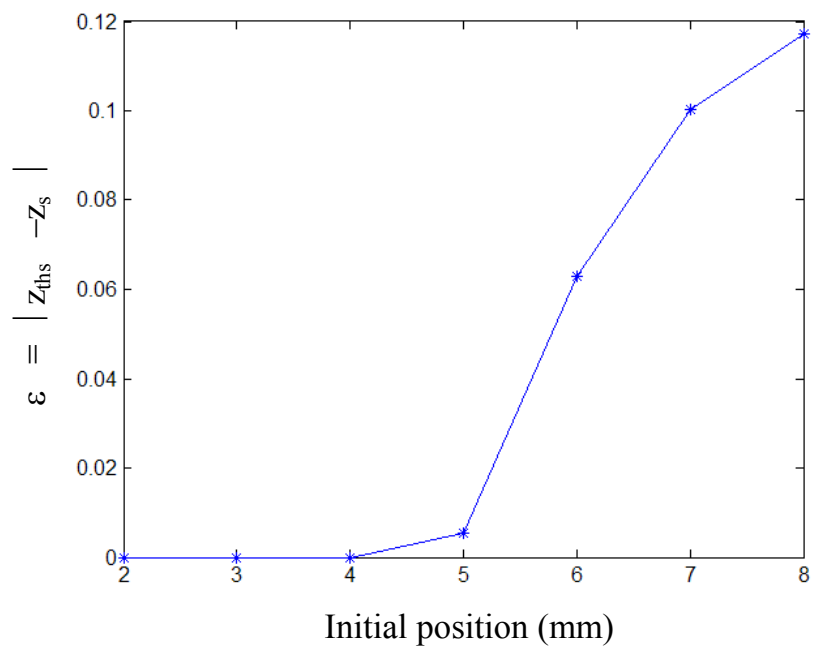


Table $1 \quad$ Alloui et al EPJ Applied Physics

TABLE 1. SAMPLE PARAMETERS

\begin{tabular}{llll}
\hline Symbol & \multicolumn{1}{c}{ Quantity } & value or expression & \\
\hline$\rho C_{p}(T)$ max & thermal capacitance $[77 \mathrm{~K} ; 300 \mathrm{~K}]$ & $-0.66+0.03 T-8.3 * 10^{-5 *} T^{2}+7.8^{*} 10^{-8 *} T^{3}\left[\mathrm{~J} \cdot \mathrm{cm}^{-3} \cdot \mathrm{K}^{-1}\right]$ & {$[17]-[18]$} \\
$\rho C_{p}(T)$ min & thermal capacitance $[77 \mathrm{~K} ; 300 \mathrm{~K}]$ & $-0.35+0.019 T-4.1 * 10^{-5 *} T^{2}+2.6^{*} 10^{-8 *} T^{3}\left[\mathrm{~J} \cdot \mathrm{cm}^{-3} \cdot \mathrm{K}^{-1}\right][17]-[18]$ & {$[19]$} \\
$\kappa$ & thermal conductivity & $6\left[\mathrm{~W} \cdot \mathrm{m}^{-1} \cdot \mathrm{K}^{-1}\right]$ & {$[19]$} \\
$T c$ & critical temperature & $92[\mathrm{~K}]$ & {$[13]$} \\
$E c$ & critical electrical field & $1[\mu \mathrm{V} / \mathrm{cm}]$ & {$[13]$} \\
$n_{0}$ & n-exponent at $77 \mathrm{~K}$ & 20 & {$[19]$} \\
$J_{c 0}$ & critical current density at $77 \mathrm{~K}$ & $440\left[\mathrm{~A} / \mathrm{mm}^{2}\right]$ & {$[13]$} \\
$h$ & convective heat transfer coefficient & $400\left[\mathrm{~W} /\left(\mathrm{m}^{2} \cdot \mathrm{K}\right)\right]$ & \\
\hline
\end{tabular}

